\title{
Théologiques
}

Théologiques

\section{Le mouton dans le trafic ou l'évangile et la ville}

\section{André Myre}

Volume 3, numéro 1, mars 1995

Symbolique urbaine et foi chrétienne

URI : https://id.erudit.org/iderudit/602417ar

DOI : https://doi.org/10.7202/602417ar

Aller au sommaire du numéro

Éditeur(s)

Faculté de théologie de l'Université de Montréal

ISSN

1188-7109 (imprimé)

1492-1413 (numérique)

Découvrir la revue

Citer cet article

Myre, A. (1995). Le mouton dans le trafic ou l'évangile et la ville. Théologiques, 3(1), 103-114. https://doi.org/10.7202/602417ar

\section{Résumé de l'article}

Qu'en est-il de la poétique évangélique, essentiellement rurale, pour qui vit en milieu urbain? L'enquête porte sur Jésus, qui évite systématiquement la ville; Paul, qui choisit systématiquement la ville; et l'évangile de Marc, selon le commentaire de Ched Myers, qui s'oppose à l'Empire de la ville de Rome et de son alliée, Jérusalem. Suivent de brèves réflexions sur la vie actuelle de disciple en ville, en situation de résistance contre l'Empire et ses alliés. d'utilisation que vous pouvez consulter en ligne.

https://apropos.erudit.org/fr/usagers/politique-dutilisation/ 


\title{
Le mouton dans le trafic ou l'évangile et la ville
}

\author{
André MYRE \\ Faculté de théologie \\ Université de Montréal
}

\section{RÉSUMÉ}

Qu'en est-il de la poétique évangélique, essentiellement turale, pour qui vit en milieu urbain? L'enquête porte sur Jésus, qui évite systématiquement la ville; Paul, qui choisit systématiquement la ville; et l'évangile de Marc, selon le commentaire de Ched Myers, qui s'oppose à l'Empire de la ville de Rome et de son alliée, Jérusalem. Suivent de brèves réflexions sur la vie actuelle de disciple en ville, en situation de résistance contre l'Empire et ses alliés.

L'imaginaire chrétien - faut-il s'en surprendre? - a été fortement influencé par les récits évangéliques, lesquels sont tout imprégnés d'une poétique rurale aux images fortes. En effet, qui a le moindrement fréquenté ces rexres ne peut oublier les oiseaux que le Père des cieux nourrir ou les lys des champs qu'il habille, le berger qui ramène sa brebis dans l'enclos, les travailleurs saisonniers appelés à amasser la récolte ou le groupe des disciples grappillant des épis en passant à travers champs. On se souvient aussi de Jean le Baptiste qui prophétise dans le désert, de Jésus qui se retire des foules quand il veut prier (quand il est à la synagogue ou au Temple, on ne dit jamais de lui qu'il prie!) ou des disciples, bâton à la main, sur les chemins poussiéreux de Palestine.

Monde rural, agricole, pastoral, autour d'un lac où se pratique la petite pêche. Monde à cent lieux de la ville moderne. Faut-il donc que le citadin chrétien se change l'imaginaire, pour l'adapter à son milieu? Une petite enquête s'impose ici, d'abord pour vérifier la justesse du souvenir, ensuite pour permettre quelques réflexions. L'enquête portera sur les rela- 
tions qu'ont entretenues avec la ville des personnages tels que Jésus, Paul et l'auteur de l'évangile de Marc.

\section{L'évangile et la ville}

\subsection{Jésus}

On sait la difficulté de tirer des évangiles des données historiques sur l'homme de Nazareth. Il ne peut donc être question ici de prouver historiquement l'attitude de Jésus par rapport à la ville. Mais la cueillette de quelques renseignements présents dans l'évangile de Marc permettra quelques conclusions plausibles, que ne contredirait pas une étude plus poussée dans les synoptiques ${ }^{1}$.

Si l'on excepte le sommaire rédactionnel de 6,56, dans lequel on dit de lui qu'il allait dans "villages, villes ou hameaux ", et 11,19 , où il sort de la ville (de Jérusalem $)^{2}$, il n'est dit des dernières années de Jésus, dans tout l'évangile de Marc, qu'à une seule reprise qu'il est dans une ville $(1,33$ : polis ), et il s'agit de Capharnaüm ( 1,21$)$. Celle-ci était une petite ville d'au plus une dizaine de milliers d'habitants à l'époque, qui n'avait rien d'une mégapole et où Jésus avait vraisemblablement sa maison ${ }^{3}$. Quand les noms des localités sont mentionnés, on dit de Jésus qu'il est à Capharnaüm $(1,21 ; 2,1 ; 9,33)$ ou Bethsaïde ${ }^{4}$. De façon surprenante, l'évangile ne nomme pas d'autre ville ou village ${ }^{5}$. On se contente de

\section{Voir la note 17.}

2 Les derniers jours de Jésus sont écartés de l'enquête, car ils sont exceptionnels en ce sens qu'ils se passent dans des lieux que Jésus avait exclus de ses engagements. Même s'il fallait modifier le paysage offert par Marc et que Jésus était allé quelques fois à Jérusalem pendant ses dernières années, il n'en reste pas moins qu'il n'y allait pas travailler.

3 Voir André MYRE, «Jésus avait-il une maison», dans Jean-Claude PETIT, André CHARRON et André MYRE, "Où demeures-tu? * La maison depuis le monde biblique. Montréal, Fides, 1994, p. 305-322.

4 La localité de Bethsaide $(8,22)$ est qualifiée de « village » au v. 23.

5 Dalmanoutha $(8,10)$ est laissé de côté, car on ne sait s'il s'agit d'une région, d'une localité, ou d'un simple point d'ancrage. 
dire que Jésus va dans sa patrie ${ }^{6}$, des villages ou hameaux ${ }^{7}$, ou, plus largement, dans une région ou un territoire ${ }^{8}$.

Entre le moment où il revient de Judée, après son baptême par Jean, et celui où il y retourne à la fin de sa vie, ses déplacements sont assez clairement situés.

- De 1,21 à 4,34, il est soit à Capharnaüm (à la synagogue, chez Simon ou André, ou à la maison ), soit aux environs ${ }^{9}$.

- Entre 4,35 et 5,43, il traverse le lac pour aller sur la rive orientale et en revient ${ }^{10}$.

- En 6,1-6a, il se rend dans sa patrie.

- Entre $6,6 b^{11}$ et 7,23 , il fait une autre traversée, au trajet un peu compliqué, vers Bethsaïde, suivie d'un retour sur la rive occidentale, à Guennèsaret et sa région, avant la rentrée "à la maison " $(7,17)$, sans doute Capharnaüm ${ }^{12}$.

- Suivent un déplacement dans le territoire païen de Tyr, puis, par Sidon ${ }^{13}$, une traversée de la Décapole $(7,24-8,9)^{14}$.

66,1 (patris) : Nazareth n'y est même pas nommé; mais en 1,9, c'est la localité d'où il vient.

7 Villages (kómé) autour de Nazareth $(6,6)$ ou de Césarée de Philippe $(8,27)$, villages en général $(6,56)$, bourgs (kómopolis) autour de Capharnaüm $(1,38)$, hameaux (agros) anonymes $(6,56)$. Ce dernier verset fait partie d'un sommaire et est rédactionnel.

8 Région (chóra) des Guéraséniens $(5,1)$ ou de Guennèsaret $(6,55)$, territoire (horion) des Guéraséniens $(5,17)$ ou de Tyr $(7,24.31)$.

9 Voir $1,21.35 .39 .45 ; 2,1.13 .15 .23 ; 3,1.7 .13 .20 ; 4,1$. Le sommaire rédactionnel de 1,39 ouvre l'activité de Jésus à « toute la Galilée », mais les traditions n'appuient pas cet élargissement.

10 Il est vraisemblable que le point de départ de 4,35 et le point de retour de 5,21 soit Capharnaüm.

11 Marc ne fait pas revenir Jésus de Nazareth; aussi est-il vraisemblable que les villages parcourus au v. 6b soient traditionnellement à situer autour de Capharnaüm.

12 Voir, pour les déplacements, 6,6b.31-32.34.45-47.51.53-56; 7,17.

13 Trajet assez inexplicable.

14 La mention que Jésus est "à la maison ", en 7,24, laisse entendre que Marc a ou bien créé ou enrichi le séjour en territoire païen, en vue de fonder la mission chrétienne en Jésus-Christ. Le parcours vers la Décapole $(7,31)$ s'est sans doute fait par mer, 
Enfin, entre 8,10 et 9,50 , Jésus et les siens vont et viennent sur la mer : de la Décapole, vraisemblablement, à la région de Dalmanoutha; nouvelle traversée vers Bethsaïde; pointe autour de Césarée de Philippe et retour à Capharnaüm ${ }^{15}$.

Qui a la curiosité de suivre ces déplacements sur une carte ne manque pas d'éprouver une certaine surprise. Évidemment, on ne sort pas de la Palestine : les grandes villes comme Alexandrie, Antioche, Athènes, ou Rome ne sont jamais même mentionnées. Mais au cours de ce qu'il est convenu d'appeler la vie publique, si on admet comme substantiellement rédactionnel son séjour en territoire païen ${ }^{16}$, Jésus ne quitte même pas la Galilée : il n'œuvre ni en Judée, ni en Samarie, ni en Haute-Galilée. De plus, si on considère comme exceptionnelles les deux pointes que forment les visites à Nazareth et à la région de Césarée de Philippe, l'espace où situer l'activité de Jésus est extrêmement restreint ${ }^{17}$. Calculé à vol d'oiseau, il s'apparente à un rectangle, situé au nord-ouest du lac de

puisque, en 8,10 , on se déplace en barque vers la région de Dalmanoutha, qui est à situer sur la rive occidentale.

15 Voir $8,10.13 .22 .27 ; 9,2.14 .28 .30 .33$. La sortie à travers la Galilée du v. 30 est sans doute rédactionnelle : $\mathrm{y}$ noter le thème très marcien du secret messianique.

16 Les données sur ce séjour tiennent en deux versers $(7,24.31)$. Quant aux trois péricopes contenues dans ce séjour, elles pouvaient fort bien avoir été situées en territoire juif, avant que l'évangéliste les utilise pour donner un contenu au voyage de Jésus.

17 L'évangile de Marc est celui qui offre le cadre narratif le plus ancien. La source $Q$ n'est somme toute qu'une suite de paroles; mais tant leur contenu (adapté à un milieu rural) que les localités mentionnées appuient les données du cadre marcien. La péricope de $L c 10,13-15 \backslash M t 11,21-24$, en particulier, est significative en ce sens qu'elle situe l'activité de Jésus à Chorazîn et Bethsaìde (ainsi que Capharnaüm), tout en niant que $T$ yr et Sidon ou Sodome aient été témoins des gestes de Jésus. En cela, $Q$ appuie la localisation marcienne de l'activité de Jésus en Galilée, mais non le déplacement dans la région de Tyr et Sidon. Jérusalem y est mentionnée, mais, sans doute en référence à la mort de Jésus, comme une ville qui tue les prophètes $(L c 13,34 \backslash M t 23,37)$. Comme Matthieu et Luc suivent substantiellement le cadre narratif offert par Marc, Jean est le seul évangile à situer une forte activité de Jésus à Jérusalem; on peut même dire que, contrairement à Marc, Jésus y œuvre surtout à Jérusalem. L'évangéliste a sans doute voulu illustrer le sérieux du conflit entre christianisme naissant et judaïsme en le portant au cœur de la ville sainte. Pour situer l'activité de Jésus, il faut donc s'appuyer sur Marc. 
Galilée, dont les dimensions sont d'environ quinze ( 15$) \mathrm{km}$ de long par huit ( 8 ) km de large ${ }^{18}$ ( voir l'illustration ci-dessous).

Légende :

1. Magdala

2. Chorazîn

3. Capharnaüm

4. Bethsaïde

5. Césarée de Philippe

6. Nazareth

7. Jérusalem
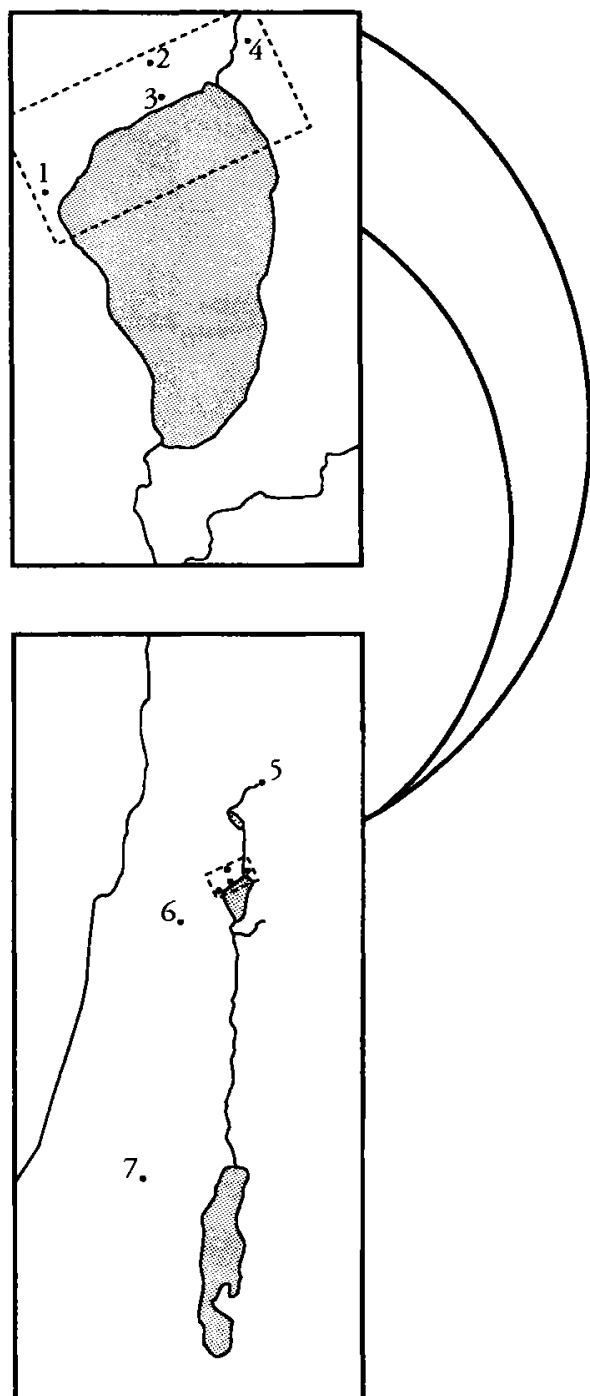

18 La base du rectangle va de Magdala à Bethsaïde, traverse le nord-ouest de la mer et permet donc de situer les fameuses traversées dont parle l'évangile. 
La ville de Capharnaüm est à peu près au centre de ce rectangle. On ne peut d'ailleurs qu'être frappé par l'importance de Capharnaüm dans l'activité de Jésus. C'est son point de départ et d'arrivée; c'est là qu'il réside; il n'en est généralement éloigné que de quelques heures de marche ou de rame ${ }^{19}$. Et c'est la seule "ville" qu'il fréquente. On ne le voit jamais entrer dans une des villes proches où la présence romaine était importante, comme Tibériade, Sepphôris ou Césarée de Philippe. Sans vouloir trop tirer d'un silence des sources, mais considérant également son absence de Jérusalem à part les derniers jours, on peut penser qu'il s'opposait à l'oppression romaine dont son pays était victime et à ceux qui s'en faisaient les complices. Opposition non violente mais quand même significative. Il avait fait le choix des petits de son peuple et se tenait loin des officiels et des lieux qu'ils fréquentaient. Il vivait son interpellation prophétique dans les marges de l'histoire, hors des grands centres, avec les marginaux. Et il évitait la "ville", qu'il s'agisse de Rome ou de Jérusalem sa complice, responsables pour une grande part de la misère des siens.

\subsection{Paul}

Pour le dire immédiatement et en quelques mots, la stratégie de Paul est exactement à l'opposé de celle de Jésus. Autant ce dernier évite les villes, autant Paul ne fréquente qu'elles 20 . Certes, il n'est pas le seul ni le

Il convient de corriger l'image traditionnelle d'un Jésus parcourant la Palestine d'une frontière à l'autre, toujours en marche et n'ayant pas de chez-soi. Ses dernières années, il les a vécues à Capharnaüm et ses environs, solidaire, avec ses disciples, des pauvres gens de son petit coin de pays.

20 Voir, sur le sujet, l'ouvrage classique de W.A. MEEKS, The first urban Christians. The social world of the apostle Paul. New Haven - London, Yale University Press, 1983. Cependant, pour avoir une vue équilibrée du christianisme naissant, il faut lire aussi G. THEISSEN, Le christianisme de Jésus, (Relais Desclée, 6), 1978. La traduction littérale du titre allemand: Sociologie du mouvement de Jésus rend mieux compte du contenu du livre que la traduction choisie par l'éditeur. THEISSEN offre un essai d'interprétation de la naissance du christianisme en Palestine, à partir de catégories sociologiques. L'auteur montre bien l'essor du christianisme dans les campagnes et son échec à Jérusalem (p. 71-83). Le parti pris pour la ville fut celui des chrétiens d'origine judéo-hellénistique et non des judéo-palestiniens, qui poursuivirent la stratégie adoptée par Jésus.

Les conflits entre les christianismes palestinien et hellénistique n'ont pas qu'une explication religieuse. La méfiance des gens de la campagne envers ceux de la ville est traditionnelle. Dans les lignes qui suivent, on présuppose que la décision de Paul 
premier à agir ainsi. Avant lui, par exemple, des communautés chrétiennes avaient été fondées à Damas ( Ac 9,19; Ga 1,17) ou Antioche ( Ac 11,1920 ). Depuis longtemps, les juifs de la diaspora étaient bien installés dans les villes hellénistiques, et les premiers convertis entreprirent tout naturellement de s'adresser à eux, avant de prendre l'initiative de se tourner vers les païens.

Paul décida lui aussi de proclamer son Évangile dans les villes de l'Empire. Il avait sans doute plusieurs raisons d'agir ainsi : il lui était plus facile de se trouver du travail et de rencontrer des gens à qui parler du $\mathrm{Christ}^{21}$, il suivait la voie tracée par ses prédécesseurs, il disposait d'un réseau de communications ( routes, courrier, etc.) bien rodé, et, peut-être surtout, le grec était la langue de communication commune aux villes. Pour évangéliser les campagnes, il aurait dû apprendre des langues, ce qui prend beaucoup de temps et entrave la mobilité. Or, pour lui, le temps pressait. Il lui fallait évangéliser les païens avant la venue du Christ. Lui faisait son travail dans les villes, à ses communautés de faire le leur dans les campagnes ( 1 Th 1,7-8).

On voit la force du contraste entre le Jésus des campagnes galiléennes et le Paul des villes hellénistiques. Or, ce contraste pose le problème de la formation des évangiles. Si on prend pour acquis, comme le fait largement l'exégèse, que les évangiles ont été écrits pour des communautés urbaines vivant après la mort de Paul, comment se fait-il qu'elles se sont reconnues dans un genre littéraire comme les évangiles, où domine tellement la poétique agricole? Simple fidélité au souvenir de Jésus? Signe d'interaction étroite entre ville et campagne? Ou faut-il réapprendre à les lire?

\subsection{Marc}

L'exégèse traditionnelle se préoccupe peu d'herméneutique. On met beaucoup d'énergie à analyser les textes, découvrir leurs structures, les situer dans l'histoire et dégager leurs niveaux de sens. Mais on n'ose pas, généralement, diriger leur force d'impact contre les institutions religieuses, sociales ou politiques contemporaines. On laisse prudemment ce

d'œuvrer dans les villes est admise; une lecture même rapide des lettres ou des Actes permet rapidement de s'en convaincre.

21 À noter que la stratégie de s'adresser aux juifs d'abord et aux païens ensuite lui est attribuée par Luc dans les Actes mais qu'on ne la trouve pas dans ses lettres. 
travail aux lecteurs ou lectrices. Et si quelqu'un s'y essaie, on fait le vide autour de son cuvre. C'est arrivé récemment à un superbe commentaire de Marc, celui de Ched Myers ${ }^{22}$, dont à peu près personne ne parle. Quelques références à cet ouvrage seront utiles à une brève réflexion sur la pertinence actuelle de l'imagerie rurale dont sont imprégnés les évangiles.

Pour Myers, l'évangile de Marc doit être lu avec un œil sur l'Empire romain ( p. 5 ), en faisant l'effort de ne pas séparer le religieux du politique, puisque la distinction ne vaut pas pour l'antiquité biblique et, peutêtre surtout, parce que cette distinction n'a servi qu'à domestiquer le premier et sacraliser le second ( $\mathrm{p}$. xxv). Il convient donc d'écouter les voix qui montent des marges sociales, celles du temps de Marc ou du nôtre, car c'est chose fondamentale pour qui veut s'éveiller à l'appel à la vie de disciple à l'intérieur de l'Empire. Mais alors que les théologiens du Tiersmonde ont l'avantage d'une certaine "affinité de site " dans leur lecture des évangiles, les résidents de l'Amérique du Nord, bien que citoyens de l'Égypte, doivent prendre le parti des Hébreux (p. 7 ). Un tel parti pris suppose la conversion par laquelle on se détourne radicalement de l'Empire, et la résistance non violente pour en ralentir les avancées. C'est en ce sens que Myers veut faire une «lecture politique " de l'évangile de Marc (p. 10).

Aux sujets de l'Empire, désireux de voir clair sur eux-mêmes et leur monde, Marc présente un chemin de disciple (p. 11 ). Il importe donc d'apprendre à bien lire son discours idéologique, si on veut défier l'infrastructure de l'Empire (p. 20 ). Pour cela, il faut apprendre à se situer au temps de Marc: "En conséquence, écrit Myers, dans tout ce commentaire, je vais parler de Marc et du Jésus de Marc, plutôt que, directement, du Jésus de Nazareth" (p.31). Quand on lit Marc, il faut toujours se demander :

22 C. MYERS, Binding the strong man. A political reading of Mark's story of Jesus. Maryknoll (NY), Orbis Books, 1988. Comme le résume bien un de ses rares recenseurs: "Il s'agit d'une lecture interdisciplinaire de l'évangile de Marc, explicitement située dans la tradition théologique de William Stringfellow, la tradition herméneutique de Norman Gottwald et la tradition exégétique marcienne de Fernando Belo» [D.M. RHOADS, CathBibQuart $53 \backslash 2$ (1991) 336]. Dans son livre, Myers annonçair un volume d'accompagnement, qui détaillerait « la forme de l'appel marcien à une vie de disciple radicale à l'intérieur de notre propre contexte " (xxvii). Il vient tout juste de paraître sous le titre: Who will roll away the stone? Discipleship queries for first-world Christians (Maryknoll (NY), Orbis Books, 1994). 
...quels sont les facteurs idéologiques impliqués dans la production d'un texte qui a la Galilée comme centre symbolique (le "pôle positif " du récit), alors que, dans le "monde réel ", ce centre est à Jérusalem (qui est le "pôle négatif» du récit!)? ... Quelle est la fonction sociale des fictions marciennes...sur les traversées de la mer en bateau,...? ... Pourquoi Jésus évite-t-il les villes,...? ( p. 35 )

Pour Myers, Marc est pour ainsi dire le seul auteur de l'Antiquité à rédiger un récit qui soit destiné au "monde ordinaire" (common people) et qui parle d'eux (p.39). Les dissidents sont au centre de son texte, et situés dans une Galilée de plus en plus aliénée. En effet, politiquement et économiquement, elle tombait sous le contrôle de la pénétration urbaine hellénistique, tandis que, tant symboliquement que socio-économiquement, elle dépendait de Jérusalem au sud (p.54). Le Jésus de Marc ne se comprend et ne peut s'interpréter qu'à l'intérieur de forts courants de résistance populaire à ces divers contrôles (p. 65). Marc est ce type de document dans lequel

...surgit un prophète qui défend une stratégie de rejet autant des Romains que de leurs collaborateurs aristocrates, et qui répudie à la fois le retrait à la Qumran et l'activisme pharisien, parce qu'ils ne s'attaquaient pas aux racines de l'oppression dans l'ordre symbolique dominant... ( p. 86 )

L'occasion de sa rédaction est l'appel aux armes, lancé en Galilée par des recruteurs rebelles, en vue de défendre Jérusalem contre l'envahisseur romain à la fin des années $60(\text { p. } 87)^{23}$.

Dans son évangile, toujours selon Myers, Marc est dur dans sa critique du Temple et de l'aristocratie parce qu'il peut se le permettre, mais il est prudent dans ses attaques contre Rome parce qu'il est en territoire occupé. Il écrit pour une communauté persécutée pour raison de subversion (p. 419 ), qui devait vivre dans la région de Caphamaüm (p. 421-423). Dans son récit, il s'oppose aux Hérodiens, fortement hellénisés, aristocratie royale concentrée dans la ville de Tibériade; aux scribes, administrateurs responsables de l'idéologie dominante; et aux anciens, grands propriétaires terriens, alliés des Romains (p.423-426). Ce sont ces gens qui refusent l'appel à la justice lancé par Jean le Baptiste $(11,27$ 33 ) et se déclarent loyaux sujets des Romains (12,13-17). Ces derniers, Marc n'hésite pas à les faire jeter symboliquement à la mer, dans les quatrième (p. 413-472) parties de son livre; le commentaire lui-même de Marc, on le comprendra, se résume difficilement. 
cochons de 5,9-13 ( p. 426-427)! Sa communauté doit vivre en marge de la société, en dehors des frontières idéologiques de l'ordre dominant, qu'il s'agisse de celles de la Judée et de son centre Jérusalem ou de celles des villes hellénistiques (p.432). Mais vivre en marge n'est pas désengagement :

Les exorcismes de Jésus, dépeints comme l'aspect le plus menaçant de sa pratique pour les autorités politiques $(3,22$ ss $)$, sont un discours par lequel Marc articule son opposition idéologique à l'hégémonie politico-militaire tant juive que romaine. L'ordre nouveau signifie la fin de la domination scribale et la libération de la botte coloniale des légions impériales. Mais l'exorcisme n'est pas que pure et symbolique déclaration d'intention: il devient très concret quand Jésus "expulse " les entrepreneurs du Temple... Cet épisode lie l'exorcisme à la politique de l'action symbolique directe (p.436).

L'évangile de Marc légitime les transgressions de l'ordre établi par le peuple des pauvres et des rejetés ( p. 439 ). Le disciple doit apprendre à vivre parmi eux et voir la vie de leur point de vue (p.440). C'est le lieu de la foi, où la prière est possible, lieu qui remplace les anciens sites sacrés ( p. 443 ), lieu de la conversion et de la résistance, y compris pour les disciples d'aujourd'hui :

...la classe moyenne nord-américaine est structurellement isolée des pauvres: c'est le sens des banlieues qui entourent nos espaces urbains,... De plus, la présence historique des pauvres est systématiquement réprimée, déformée ou objectivée dans les médias impériaux : c'est le sens de Hollywood, des journaux télévisés de $18 \mathrm{~h} 00$ et de la presse qui s'autocensure. ... une authentique solidarité avec les opprimés ne conduit pas à une satisfaction vicariale, mais à une douloureuse rencontre avec nos moi impériaux... ( p. 451 )

Le commentaire de Myers nous fair plonger dans la vie. Pour le lire adéquatement, il faut avoir en tête et savoir distinguer trois lieux culturels différents : celui de Jésus, celui de Marc et le nôtre (en Amérique du Nord ). Sa lecture de Marc situe le lecteur au cœur des enjeux impériaux, des compromissions des autorités en place, des espoirs de changement par la violence. En surface, la poétique est rurale, au fond, les préoccupations sont urbaines. Rome et Jérusalem, Tibériade, Sepphôris ou Césarée de Philippe sont constamment dans la ligne de tir, mais l'action est ailleurs, sur la mer, qui est l'Empire qu'on exorcise, dans les villages, les hameaux et les champs, qui sont là où n'est pas l'Empire. Le salut est dans les marges de la société, avec le peuple des pauvres, dans le refus de l'idéologie ( religieuse, sociale ou politique) dominante. Mais il est inévitable 
que meure d'abord celui qui espère ce salut, assassiné par l'Empire, et sans que Dieu fasse quoi que ce soit, dans tout l'évangile, à part de reconnaître son fils. Une chose est sûre, cependant, pour les disciples comme pour Jésus, le salut de Dieu ne peut se rencontrer dans la ville de Jérusalem, l'alliée de la ville de Rome. Il faut partir pour la Galilée ( Mc 16,7), où se situe la résistance des pauvres.

\section{Le mouton dans le trafic}

Le commencement de la Bonne Nouvelle, selon Marc, c'est que Jean est au désert et que Jésus vient le rencontrer. Le récit débute dans les marges de l'Empire, à sa périphérie. Rome tout comme Jérusalem sont exclues des événements déterminants de l'Histoire. L'important ne se passe pas en ville. Et la suite, en Galilée, le démontrera. Or, de façon paradoxale, la Galilée de jadis, c'est aujourd'hui, pour une large part, la ville.

À une époque où l'Empire s'étend partout, dans les banlieues, à la campagne, autour des lacs, sur les plages, avec ses aéroports, ses terrains de golf, ses centres d'achat, ses complexes financiers et sportifs ou ses chalets, il laisse la plus grande partie de la ville aux pauvres. Les riches en argent fuient la ville ou s'enferment dans leurs forteresses. Les riches en vie spirituelle ou en convictions écologiques sortent de la ville pour aller prier à la campagne, nouveau site sacré. Ils laissent au peuple des pauvres la chaleur ou le froid, le béton, l'entassement, le délabrement. C'est à la ville qu'on trouve les "foules", les "païens", la violence, l'exploitation. L'évangile appelle à vivre en ville, dans ces espaces de la ville dont l'Empire est absent ou qu'il quitte à $17 \mathrm{~h} 00$.

L'Empire a des alliés, nouveaux "scribes, anciens, Hérodiens, pharisiens". Ordinairement, le peuple des pauvres ne rencontre pas directement l'Empire, mais ses représentants : technocrates, fonctionnaires, voix, anonymes ou pas, réclamant le paiement des comptes, annonçant démolitions ou rénovations, coupures ou rajustements, démotions ou renvois. Gens souvent de bonne volonté, mais avec qui les défenseurs des groupes populaires ou les disciples ont d'ordinaire leurs démêlés.

Les alliés de l'Empire sont, fréquemment aussi, gens de religion. Fils de colonisateurs, ils refusent, qu'en Amérique Latine, des peuples jadis soumis se libèrent ou développent une pensée qui menace l'hégémonie de la leur. Hommes sûrs d'eux-mêmes, ils n'acceptent pas que d'autres anciennes colonies, en Amérique du Nord, fassent place égale aux femmes. Blancs supérieurs, ils refusent que l'Afrique jadis esclave devienne Église sœur. Ils négocient d'État à État avec l'Empire, seuls habi- 
lités à le faire, pendant que les disciples se font tuer ou écraser. Et ils obligent leurs fonctionnaires à se former et à vivre séparés des foules pour lesquelles ils existent. C'est une bien étrange chose que les disciples doivent se méfier de ceux-là mêmes, les bergers, qui sont chargés de les protéger de l'Empire.

Le peuple des pauvres, nuisance aux yeux de l'Empire, est dans la ville. C'est là que lui sont aujourd'hui envoyés les disciples, deux par deux, toujours en mouvement, déménageant fréquemment pour suivre les leurs, capables de le faire parce que vivant sans être trop encombrés de biens, utilisant le transport en commun comme les foules. C'est là qu'ils multiplient les pains, font trouver un logement, défendent contre un fonctionnaire ou un violent, fraternisent avec le peuple des pauvres venu d'ailleurs, aident à porter l'insécurité, accompagnent dans la maladie et la solitude, manifestent contre l'Empire et ses alliés.

C'est dans la ville aussi qu'ils vivent, avec les leurs. En petits réseaux dispersés. Dans une certaine clandestinité qu'impose la vie dans les marges de la société. L'Empire et ses alliés se méfient, il ne sert à rien de les alerter. Il serait dangereux de monter à Jérusalem trop souvent. Mais les rencontres sont nécessaires, où le pain se partage, où la fraternité et la confiance s'expérimentent, où les relations hommes-femmes se vivent dans l'égalité, où le pouvoir, même sacré, est détruit, où la possibilité d'aller jusqu'au bout de soi se creuse.

Cette vie de disciple est présentement chose aussi petite, en ville, que la vie de Jésus jadis, en Galilée. Mais ce n'est pas sa poétique rurale qui fait écran à l'évangile dans nos sociétés urbaines. C'est que les villes sont bondées, et que les disciples sont peu nombreux. L'Empire est puissant et ses alliés empressés. Les besoins humains sont pressants, et les scribes racontent des paraboles. La misère augmente, et les pauvres en sont rendus responsables. Les pécheurs abondent, et on leur parle d'un Dieu pour les purs. Jésus était subversif, et on présente un conformiste. Il critiquait les institutions, et on lui en fait fonder une, immuable. La contestation de l'évangile est presque insoutenable, et on l'apprivoise à outrance. La poétique rurale de l'évangile apparaît souvent dépassée parce qu'on y a réduit l'évangile ou encore domestiqué sa force subversive.

Il faut relire et relire l'évangile pour en retrouver l'impact et oublier définitivement le petit Jésus rosé, blond et frisé ramenant son mouton blanc dans l'enclos. Le mouton de l'évangile réclame aujourd'hui sa part d'investissements en Afrique, la fin de sa dette en Amérique Latine, l'abolition du nettoyage ethnique en Europe, l'égalité homme-femme en Amérique. Le mouton est dans le trafic. Et, souvent, il en meurt. 